\title{
Evaluation of heterosis and its association among morpho-physiological traits of ten wheat genotypes under water stress
}

Nazeer Ali Panhwar ${ }^{1 *}$, Gul Muhammad Baloch ${ }^{1}$, Zahoor Ahmed Soomro ${ }^{1}$, Mahboob Ali Sial ${ }^{2}$, Sajjad Ali Panhwar ${ }^{3}$, Ambreen Afzal ${ }^{4}$ and Altaf Hussain Lahori ${ }^{5}$

1. Department of Plant Breeding and Genetics, Sindh Agriculture University Tandojam, 70060, Pakistan

2. Division of Plant Breeding and Genetics, Nuclear Institute of Agriculture Tandojam, 70060, Pakistan

3. Department of Crop Genetics and Breeding Hainan University Haikou City, 570206, China

4. Department of Geography, University of Karachi, 75270, Pakistan

5. Department of Environmental Sciences, Sindh Madressatul Islam University, Karachi 74000, Pakistan

*Corresponding author's email: nazir_ipanhwar@yahoo.com

Citation

Nazeer Ali Panhwar, Gul Muhammad Baloch, Zahoor Ahmed Soomro, Mahboob Ali Sial, Sajjad Ali Panhwar, Ambreen Azfal and Altaf Hussain Lahori. Evaluation of heterosis and its association among morpho-physiological traits of ten wheat genotypes under water stress. Pure and Applied Biology. Vol. 11, Issue 3, pp709-724. http://dx.doi.org/10.19045/bspab.2022.110072

Received: 09/08/2021 Revised: 22/09/2021

Accepted: 24/09/2021

Online First: 07/10/2021

\section{Abstract}

Wheat is a staple food grain ranked that first as consumed globally and occupies a central position in the cereal trade. Grain yield is a polygenic inherited trait and is the product of several attributes which contribute to it direct or indirectly. Wheat production is affected by various calamities, among them; water stress is a destructive factor to the seed yield of wheat. In present study, we investigated the performance of $F_{1}$ wheat hybrids for heterosis and the relation between morpho-physiological characters under water stress at field conditions. Split plot design was used consisted of two factors; genotypes and water stress with three replications. Ten $F_{1}$ wheat hybrids generated using half-diallel crossing and were evaluated under water stress treatments viz., normal watering $\left(\mathrm{T}_{0}\right)$, extreme water stress from tillering to maturity $\left(\mathrm{T}_{1}\right)$, and post-flowering water stress from anthesis to maturity $\left(\mathrm{T}_{2}\right)$. The obtained results revealed that the $\mathrm{F}_{1}$ wheat hybrids Sarsabz x Kiran-95 (V7), TD-1 x NIA-Sarang (V8) and NIA-Sarang x Kiran-95 (V10) were found potential wheat hybrids for seed yield under both water stress and normal watering which could be included in the future wheat breeding scheme. Hybrid performance during the wheat breeding scheme can be improved by analyzing genetic potential among wheat genotypes, determining the association between traits and selection with greater genetic diversity and heterosis for water stress tolerance and identification of traits most influenced grain yield. Overall these newly evolved hybrids should be recommended for cultivation under normal field conditions.

Keywords: Correlation; $\mathrm{F}_{1}$ hybrid; Heterosis; Morpho-physiological traits; Triticum aestivum L; Water stress

\section{Introduction}

Wheat is an important staple food cereal ranks first among cereals in the world, generally known as the king of cereals for acreage it occupies, high production and major status in trade of international food grain market [1, 2]. Rapidly increasing human population has changed climatic 
conditions and showed concerns to global food security [3]. The current improvement of several important crops is inadequate to meet future food demand [4]. Wheat is an annual, autogamous rabi crop, belongs to the tribe triticeae, and is hexaploid with AA BB DD genome. World production of wheat is being affected by environmental calamities among them water scarcity has been considered a huge problem. Grain yield is a polygenic inherited trait and is the product of several attributes which affect it direct or indirectly [5]. For genetic manipulation, it is necessary to create genetic variability for improving yield and yield-associated attributes [6]. Sufficient genetic variation is necessary for crop improvement schemes, while the presence of variability among wheat genotypes for yield and yield related components was reported by $[7,8]$.

A successful wheat breeding program needs genetic diversity as a prior condition and of paramount importance in the modern sustainable agriculture. Parents can produce a hybrid with better yield performance. Hence clear information on the nature, pattern and degree of genetic diversity help wheat breeders to choose diverse parents for hybridization [9, 10]. Asifa et al. [11] revealed that drought tolerance wheat species are based on morpho-physiological traits. Bernardo et al. [12] examined the metabolic responses triggered by arbuscular mycorrhiza increase survival potential to moisture stress in wheat genotypes. Lin et al. [13] revealed the heterosis-related genes confer bumper production in super hybrid rice. Moosavi et al. [14] investigated the phenological, morphological, physiological, and proteomic traits of Triticum boeticum under drought stress conditions. Therefore, this research study aimed to investigate the $F_{1}$ wheat hybrids for heterosis and the relation between morpho-physiological characters under three water stress field conditions. It is hypothesized that the morpho-physiological characters of $F_{1}$ wheat hybrids for heterosis might be affected under water stress conditions.

\section{Materials and Methods}

This research was performed at the field of Division of Plant Breeding and Genetics, Nuclear Institute of Agriculture (NIA) Tandojam, as shown in (Fig. 1) study area. A split plot design was used consisted of two factors; genotypes and water stress with three replications. Genotypes were sub plots; ten $\mathrm{F}_{1}$ wheat hybrids were included with their five parents. Main plots were treated with three water treatments viz., normal watering $\left(\mathrm{T}_{0}\right)$, extreme water stress from tillering to maturity $\left(\mathrm{T}_{1}\right)$ and post flowering water stress from anthesis to maturity $\left(\mathrm{T}_{2}\right)$. Half diallel design was used followed by Griffing's method-2, model-1 a numerical approach; $\mathrm{n}(\mathrm{n}-1) / \mathrm{n}$. F 1 wheat hybrids; T J-83 x Sarsabz, T J-83 x TD -1, T J-83 x NIA- Sarang, TJ-83 x Kiran-95, Sarsabz x TD-1, Sarsabz x NIASarang, Sarsabz x Kiran-95, T D-1 x NIASarang, T D-1 x Kiran-95, and NIA-Sarang x Kiran-95. Parents cultivars included TJ-83, Sarsabz, TD-1, Kiran-95 and NIA-Sarang were grown along with their hybrids. Seeds were dibbled. The middle two rows were used to select randomly ten plants of each sub plot. In addition, the recommended dose of nitrogen $(\mathrm{N})$ in the form of urea was applied at $120 \mathrm{~kg} \mathrm{ha}^{-1}$ and Phosphorus (P) in the form of DAP was incorporated at $75 \mathrm{~kg} \mathrm{P}_{2} \mathrm{O}_{5} \mathrm{ha}^{-1}$. All management practices were uniformly applied. Four rows of $2.5 \mathrm{~m}$ length with $30 \mathrm{~cm}$ and $15 \mathrm{~cm}$ distance between rows and plants respectively were maintained.

\section{Morpho-physiological traits}

Days to $50 \%$ flowering, days to $90 \%$ maturity, plant height, main spike length, tillers plant ${ }^{-1}$, spikelet spike ${ }^{-1}$, grains spike ${ }^{-1}$, 1000 grain weight, grain yield plant $^{-1}$, biological yield ${ }^{-1}$, harvest index, flag leaf area, leaf relative water content, and chlorophyll content. Furthermore, days to $50 \%$ flowering were noted from sowing date 
to date of $50 \%$ flowering of individual genotype. Days to $90 \%$ maturity were noted from date of sowing to date of visually $90 \%$ maturity of individual genotype. Plant height was measured in centimeters from the surface of the soil to the tip of the spike. The main spike length was noted in centimeters from the base of the rachis to the tip of a spike in a field standing crop. The fertile tillers of each genotype were noted at the time of 'maturity in the field standing crop. The number of spikelet spike ${ }^{-1}$ of each genotype was noted in the laboratory. The number of grains spike
${ }^{1}$ was counted after hand threshing the spike in the laboratory. Seed index was noted by weighing 1000 grain. Grain yield plant ${ }^{-1}$ was noted by weighing the total grains of a plant. The biological yield was noted by weighing uprooted plants before threshing. Harvest index was analyzed as grain yield/biological yield $x$ 100. The flag leaf area was measured in the laboratory of the Division of Plant Breeding and Genetics at NIA Tandojam using portable laser leaf area meter AG-51020, connected with a high speed scanner and scan board/data logger.

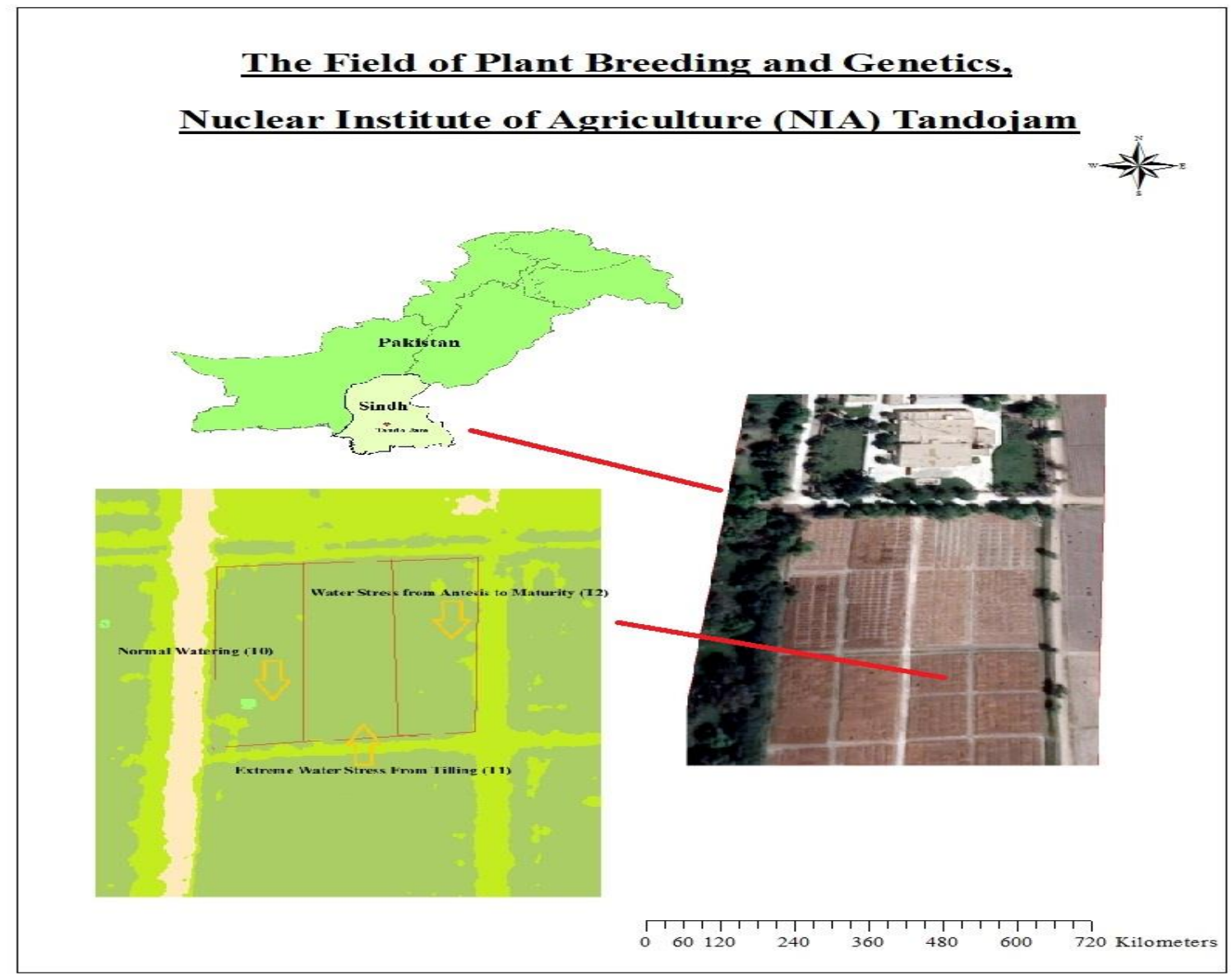

Figure 1. Study Area; The field of division of plant breeding and genetics, Nuclear Institute of Agriculture (NIA) Tandojam

\section{Leaf relative water content}

Leaf relative water content was analyzed to cut fully expanded leaf at the second node from the top of canopy to note relative water content in Laboratory where initial weight, turgid weight and dry weight was noted to assess the impact of an increase in the intensity of water stress on physiological 
parameters. Leaves were cut from adjoining point of leaf lamina and leaf sheath and taken to the laboratory immediately to prevent water loss. Fresh weight was determined on the top loading digital weight balance. This foliage was then separately kept into deionized water in a test tube for $24 \mathrm{hr}$ in a cold room for rehydration. Each test tube contained a leaf from a single genotype and replication. After $24 \mathrm{hr}$, leaves were then used for noting turgid weight. Then carefully placed in a small paper bag and oven dried at $72^{\circ} \mathrm{C}$ for $24 \mathrm{hr}$ to examine the dry weight of leaves. Relative water content was determined followed by Baars and Weatherley [15] as;

Fresh weight- Dry weight/Turgid weight x10 Chlorophyll content

Chlorophyll content was obtained from leaf $2^{\text {nd }}$ of the flag leaf. Three readings were noted with chlorophyll meter M-52 from the base, mid and near the end of leaf lamina. 1

\section{Soil physico-chemical analysis}

In this study, before planting of wheat soil was tested by drawing soil samples randomly which were collected from $0,6 \mathrm{~cm}$ and $15 \mathrm{~cm}$ at the depth. In addition, soil texture was analyzed by the Bouyoucos Hydrometer method [16]. Soil field capacity was measured according to Veihmeyer and Hendrickson [17]. Soil organic matter was determined by the Walkley-Black method [18]. Soil EC and $\mathrm{pH}$ were measured in 1:5 $\mathrm{H}_{2} \mathrm{O}$ (W/V) according to Kwon et al. [19]. Total nitrogen (TN) was detected via Kjeldahl protocol (USEPA Method 351.2). Total phosphorus $(\mathrm{P})$ and potassium $(\mathrm{K})$ were determined by $\mathrm{NaOH}$ fusion procedure [20]. The studied soil was sandy loam in texture, field capacity $13 \%$ by weight, organic matter $0.97 \%$, EC $2.7 \mathrm{mMolS}^{-1}, \mathrm{pH} 7.4$, nitrogen $0.61 \mathrm{~g} \mathrm{~kg}^{-1}$, phosphorus 19.8ppm and potassium 140ppm, were measured at the Soil science laboratory of Nuclear Institute of Agriculture Tando Jam.

\section{Heterosis}

Heterosis is the performance of $F_{1}$ hybrids over their parents. Comparative heterosis was analyzed according to Baloch et al. [21]. Increase and decrease ratio over mid and better parent was computed as; F1-MP /MP x 100 and F1-BP /BP x 10

\section{Pearson correlation coefficient}

Coefficient of correlation between some morpho-physiological characters was computed followed by Baloch et al. [22] as; Coefficient of Correlation $=$ Covariance $/$ Geomet-ric mean of covariance.

$$
\sum X Y+\Sigma x i_{-}{ }^{2}=\Sigma X^{2}-(\Sigma X)^{2} / \mathbf{N}=\Sigma y^{2}=\Sigma X^{2}-(\Sigma X)^{2} / \mathbf{N}
$$

(Where; $\mathrm{X}=$ Independent variable, $\mathrm{Y}=$ Dependent variable, $\mathrm{N}=$ Number of observations).

\section{Geospatial techniques}

Geospatial techniques are remarkable in the field of research for managing, monitoring huge data set easily and efficiently. In this research, high-resolution satellite imaginary data have been extracted from google earth then geo-referencing techniques have been applied for image rectification. For extraction of the area of interest, a mask tool has been used. The editor tool has been used for the digitization of image data.

\section{Statistical analysis}

The obtained data were subject to statistical analysis of variance by using Statistics software version 8.1 as suggested by Steel and Torrie [23].

\section{Results and Discussion}

As shown in (Table 1) the mean squares indicated that there were highly significant modifications among water treatments. Highly significant differences $(\geq 0.01 \%)$ between genotypes under water treatments were noted for most of the characters except 
the flag leaf area. Interaction between genotype and treatment showed highly significant variations for most of the characters except tiller plant ${ }^{-1}$ and flag leaf area. It was noticed from (Table 1) that treatments, genotypes, and their interaction showed differently for the morphophysiological character. Similarly, Sial et al. [10] found significant influences of water deficit on seed production and yield related characters of wheat populations.

Table 1. Mean square of morpho-physiological traits of $F_{1}$ wheat hybrids and their parents.

\begin{tabular}{|c|c|c|c|c|c|c|}
\hline \multirow[b]{2}{*}{ Characters } & & \multicolumn{5}{|c|}{ Mean squares } \\
\hline & $\begin{array}{c}\text { Replicatio } \\
\text { n D.F }=2\end{array}$ & $\begin{array}{c}\text { Treatmen } \\
\text { tD.F }=2\end{array}$ & $\begin{array}{c}\text { Repli } x \\
\text { Treat } \\
\text { D.F=4 }\end{array}$ & $\begin{array}{c}\text { Genotype } \\
\text { D.F }=14\end{array}$ & $\begin{array}{c}\text { Treat } x \\
\text { Genot } \\
\text { D.F=28 }\end{array}$ & $\begin{array}{c}\text { Error } \\
\text { D.F }=56\end{array}$ \\
\hline Days to flower & 113.3 & $35753.5 * *$ & 13.9 & $50.1 * *$ & $52.01 * *$ & 3.3 \\
\hline Days to maturity & 0.67 & $5281.25 * *$ & 0.49 & $34.56 * *$ & $92.57 * *$ & 2.08 \\
\hline Plant height & 3.51 & $4657.81 * *$ & 5.05 & $210.02 * *$ & $74.75^{*}$ & 16.4 \\
\hline Tillers plant $^{-1}$ & 20.58 & $87.38 * *$ & 4.03 & $10.45 *$ & $3.74 \mathrm{~ns}$ & 1.88 \\
\hline Spike length & 0.25 & $161.5 * *$ & 0.16 & $2.93 * *$ & $2.2 * *$ & 0.19 \\
\hline Spikeletspike $^{-1}$ & 0.51 & $167.49 * *$ & 2.29 & $4.86 * *$ & $3.96^{* *}$ & 0.55 \\
\hline Grainsspike $^{-1}$ & 110.18 & $23.84 * *$ & 2.09 & $218.93 * *$ & $193.89 * *$ & 17.42 \\
\hline $\begin{array}{c}\text { Grain } \\
\text { yieldmainSpk }\end{array}$ & 1.08 & $12.55 * *$ & 0.91 & $0.52 * *$ & $0.46^{*}$ & 0.05 \\
\hline 1000 grain weight & 1.51 & $233.07 * *$ & 3.1 & $63.43 * *$ & $70.66^{* *}$ & 1.83 \\
\hline Biol. yield plant ${ }^{-1}$ & 63.9 & $21107.6^{* *}$ & 1.2 & $448.9 * *$ & $888.9 * *$ & 5.1 \\
\hline Grain yield plant $^{-1}$ & 3.46 & $6102.69 * *$ & 16.21 & $104.59 * *$ & $122.63 * *$ & 6.55 \\
\hline \multirow[t]{2}{*}{ Harvest index\% } & 0.1 & $33920.4 * *$ & 0.1 & $46.09 * *$ & $47.3 * *$ & 1.0 \\
\hline & \multicolumn{6}{|c|}{ Physiological characters } \\
\hline Flag leaf area $(\mathrm{mm})$ & 830619 & $2.351 \mathrm{~ns}$ & 1.522 & $1.676 \mathrm{~ns}$ & $1.083 \mathrm{~ns}$ & 3.98574 \\
\hline $\begin{array}{c}\text { Relative water } \\
\text { content }\end{array}$ & 397.78 & $841.31 * *$ & 2.135 & $260.13 * *$ & $119.59 * *$ & 1.6772 \\
\hline Chlorophyll content & 9.82 & $202.37 * *$ & 1.02 & $22.96^{*}$ & $29.19 * *$ & 3.15 \\
\hline
\end{tabular}

$*=$ Significant at $0.5 \%$ level of probability, $* *=$ significant at $0.1 \%$ level of probability, ns=non-significant

\section{Plant height}

Heterotic performance of $\mathrm{F}_{1}$ wheat hybrids for plant height (Table 2) under normal watering $\left(\mathrm{T}_{0}\right)$ indicated that TJ-83 x TD-1 ($14.10,-19.00)$ showed the shortest stature of all the $F_{1}$ hybrids against their mid and better parent. Other short statured hybrids were TJ83 x NIA-Sarang $(-6.45,-6.50)$ and TJ-83 x Sarsabz (-0.15, -1.40) against their mid parent (MP) as well as the better parent (BP). For the selection of short statured wheat genotypes, these hybrids could be included in breeding schemes. The remaining wheat hybrids could be included in the selection scheme for high kernel and biological yield. The (Table 2) revealed that TD-I x Kiran-95
$(-0.30,-5.40)$ showed a reduction in plant height beside both its MP with BP. TD-1 x NIA-Sarang $(2.00,-3.00)$ and Sarsabz $x$ Kiran-95 (2.20,-0.5) showed dwarf plants against their BP. Only wheat hybrid TD-1 x NIA-Sarang (-2.25, -7.20) showed decreased plant height beside both MP with BP under water deficit $\left(\mathrm{T}_{2}\right)$. The remaining all hybrids observed an increased plant height besides their both MP and BP under the same water stress. Sial et al. [10] also reported significantly reduced plant height under severe water stress.

\section{Main spike length}

The data in (Table 2) revealed that all genotypes found an increased main spike 
length besides both MP and BP under $\left(\mathrm{T}_{0}\right)$. The large main spike was observed by $F_{1}$ wheat hybrid TD-I x NIA-Sarang $(6.95,6.70)$ as compared to Sarsabz x TD-I (6.70, 6.30), Sarsabz x Kiran-95 (6.55, 5.50), NIA-Sarang $x$ Kiran-95 (6.50, 6.50), Sarsabz x NIASarang (6.25, 5.60), TD-I x Kiran-95 (5.45, 4.80) and TJ-83 x Sarsabz (5.40, 3.80) respectively against their $\mathrm{MP}$ and $\mathrm{BP}$ under normal watering $\left(\mathrm{T}_{0}\right)$. Water stress from tillering to maturity $\left(T_{1}\right)$ influenced the main spike length of $F_{1}$ wheat hybrids. Hybrid Sarsabz x NIA-Sarang (1.80, 1.50), TD-I x Kiran-95 (1.40, 0.40), and NIA-Sarang $x$ Kiran-95 (0.80, 0.70) surpassed their MP, as well as BP concerning the main spike length and rest of hybrids, were reduced in main spike length. The largest spike length of all wheat hybrids was noted in $\mathrm{F}_{1}$ hybrid Sarsabz $\mathrm{x}$ NIA-Sarang $(4.75,4.20)$ as compared to TJ-83 x NIA-Sarang $(3.75,2.20)$, NIASarang x Kiran-95 (3.25, 1.20), Sarsabz x TD-I (2.90, 1.40), Sarsabz x Kiran-95 (2.80, 1.30), TD-I x NIA-Sarang $(2.75,0.70)$ and TJ-83 x Sarsabz $(2.40,1.40)$ respectively over their MP as well as BP. This material could be utilized for a selection of large main spike wheat genotypes. Sharma [24] reported that in the hybridization system, viz., biparental mating and diallel selective mating, which develop both functional and nonfunctional gene influences, at the same time, could be beneficial in the step-up of spike length in durum wheat.

Table 2. Heterotic effects of $F_{1}$ wheat hybrids on plant height and main spike length under water treatments

\begin{tabular}{|c|c|c|c|c|c|c|c|c|c|c|c|c|}
\hline \multirow{3}{*}{ Genotypes } & \multicolumn{6}{|c|}{ Plant height } & \multicolumn{6}{|c|}{ Main spike length } \\
\hline & \multicolumn{2}{|c|}{$\begin{array}{c}T_{0}(\text { Control }) \\
\text { I/D \% }\end{array}$} & \multicolumn{2}{|c|}{$\begin{array}{c}\mathbf{T}_{1} \\
\mathrm{I} / \mathrm{D} \%\end{array}$} & \multicolumn{2}{|c|}{$\begin{array}{c}\mathbf{T}_{2} \\
\mathbf{I} / \mathbf{D} \%\end{array}$} & \multicolumn{2}{|c|}{$\begin{array}{c}\mathbf{T}_{0} \\
\text { I/D \% }\end{array}$} & \multicolumn{2}{|c|}{$\begin{array}{c}\mathrm{T}_{1} \\
\text { I/D } \%\end{array}$} & \multicolumn{2}{|c|}{$\begin{array}{c}\mathbf{T}_{2} \\
\mathbf{I} / \mathbf{D} \%\end{array}$} \\
\hline & MP & $\mathbf{B P}$ & MP & $\mathbf{B P}$ & MP & BP & MP & $\mathbf{B P}$ & MP & $\mathbf{B P}$ & MP & $\mathbf{B P}$ \\
\hline V1 & -0.15 & -1.40 & 6.08 & 7.80 & 10.85 & 2.90 & 5.40 & 3.80 & -2.55 & -4.00 & 2.40 & 1.40 \\
\hline $\mathrm{V} 2$ & -14.10 & -19.00 & 10.15 & 8.20 & 2.90 & 1.30 & 4.60 & 3.40 & -3.15 & -3.80 & 1.80 & 1.30 \\
\hline V3 & -6.45 & -6.50 & 14.25 & 7.30 & 6.55 & 0.00 & 4.05 & 3.10 & -3.65 & -5.40 & 3.75 & 2.20 \\
\hline V4 & 2.05 & 1.20 & 5.65 & -1.40 & 13.90 & 8.50 & 4.15 & 3.60 & -2.95 & -4.60 & 1.30 & 0.80 \\
\hline V5 & 11.75 & 8.10 & 12.30 & 9.90 & 13.35 & 7.00 & 6.70 & 6.30 & 0.00 & -0.80 & 2.90 & 1.40 \\
\hline V6 & 4.40 & 3.20 & 7.30 & 4.70 & 2.70 & 1.30 & 6.25 & 5.60 & 1.80 & 1.50 & 4.75 & 4.20 \\
\hline V7 & 7.40 & 5.30 & 2.20 & -0.50 & 13.55 & 11.00 & 6.55 & 5.50 & -3.50 & -3.70 & 2.80 & 1.30 \\
\hline V8 & 9.25 & 4.40 & 2.00 & -3.00 & -2.25 & -7.20 & 6.95 & 6.70 & 0.90 & -0.20 & 2.75 & 0.70 \\
\hline V9 & 1.45 & -4.30 & -0.30 & -5.40 & 4.40 & 0.60 & 5.45 & 4.80 & 1.40 & 0.40 & 1.40 & 1.40 \\
\hline V10 & 13.10 & 12.20 & 7.30 & 7.20 & 14.30 & 10.50 & 6.50 & 6.50 & 0.80 & 0.70 & 3.25 & 1.20 \\
\hline
\end{tabular}

Note: $\mathrm{MP}=$ Mid parent, $\mathrm{BP}=$ Better parent, I= Increase, $\mathrm{D}=$ Decrease; $\mathrm{V} 1=\mathrm{TJ}-83 \times$ x Sarsabz, V2= TJ-83 x TD-1, V3=TJ-83 x NIA-Sarang, V=TJ-83 x Kiran-95, V5= Sarsabz x TD-1, V6=Sarsabz x NIA-Sarang, V7=Sarsabz x Kiran-95, V=8 TD-1 x NIA-Sarang, V9 TD-1 x Kiran-95 and V10=NIA-Sarang x Kiran-95

\section{Chlorophyll content}

Chlorophyll content is the important physiological character of a plant and generally called the blood of the plant. Chlorophyll plays important role in the manufacture of starch. Water stress directly affects chlorophyll pigmentation which ultimately influences the end product of the plant. According to (Table 3) wheat hybrids transgressed over MP as well as BP concerning chlorophyll concentration under
$\left(\mathrm{T}_{0}\right)$. Great chlorophyll concentration was noted in hybrid TD-1 x NIA Sarang (11.30, 8.40) over MP and BP as compared to TJ-83 x Kiran-95 (9.10, 8.40), NIA-Sarang x Kiran95 (8.15, 6.50), Sarsabz x TD-1(7.50, 6.00), Sarsabz x Kiran-95 (4.75, 4.50), TJ-83 x TD1 (4.35, 3.80), Sarsabz x NIA-Sarang (3.70, 2.30) and TD-1 x Kiran-95 (2.75, 1.50) respectively under normal watering $\left(\mathrm{T}_{0}\right)$. According to (Table 3) the $\mathrm{F}_{1}$ wheat hybrid such as Sarsabz x NIA-Sarang (7.90, 7.40) 
showed great chlorophyll content over mid as well as a better parent, followed by TJ-83 x TD-1 (5.80, 4.30), TD-1 x Kiran-95 (4.95, 3.80), Sarsabz x TD-1 (4.80, 3.40), and TJ-83 x Sarsabz $(1.50,1.40)$ were surpassed for chlorophyll content against their mid as well as BP under $\left(\mathrm{T}_{1}\right)$. The wheat hybrid such as Sarsabz x NIA-Sarang $(6.15,4.50)$ surpassed $\mathrm{MP}$ as well as BP for chlorophyll content, followed by TD-1 x Kiran-95 (6.80, 3.50), TD-1 x NIA-Sarang (3.50, 3.30), Sarsabz x TD-1 (3.05, 1.20) and TJ-83 x Kiran-95 $(2.80,0.80)$ respectively surpassed to their MP as well as BP for chlorophyll content.

\section{Flag leaf area}

Flag leaf adds food manufacturing in plants up to maturity and is an important source to sink. The larger area of flag leaf would capture more solar light as compared to smaller. Results in (Table 3) revealed that hybrids showed increased flag leaf area against their MP as well as BP under normal watering $\left(\mathrm{T}_{0}\right)$. The large flag leaf of all hybrids was attained by $\mathrm{F}_{1}$ wheat hybrid TDI x NIA-Sarang $(117.10,69.90)$ as compared to Sarsabz x NIA-Sarang $(76.90,60.40)$, NIA-Sarang $x$ Kiran-95 (73.90, 55.3),
Sarsabz x Kiran-95 (50.70, 15.06), Sarsabz x TD-1 (37.80, 7.10), TJ-83 x Sarsabz (36.75, 8.30) and TJ-83 x Kiran-95 (19.45, 12.8) which surpassed to their MP as well as BP. However, $F_{1}$ wheat hybrid TD-I x NIASarang $(120.65,81.8)$ showed the largest flag leaf area of all hybrids, as well as MP and BP, followed by NIA-Sarang x Kiran-95 (88.95, 80.60), Sarsabz x NIA-Sarang (82.40, 59.30), Sarsabz x TD-1 (81.45, 74.00), TD-1 x Kiran-95 $(52.3,21.8)$ and Sarsabz x Kiran-95 $(45.45,21.70)$ which also showed increased flag leaf area against their MP and BP under same water stress. The wheat $\mathrm{F}_{1}$ hybrid TD-1 $x$ NIA-Sarang $(116.80,35.90)$ was noted with the highest flag leaf area overall hybrids and its MP as well as BP. TD-1 x Kiran-95(89.65, 36.30), Sarsabz x TD-1 (76.20, 7.8), TJ-83 x Sarsabz (65.40, 53.60), Sarsabz x Kiran-95 $(28.95,13.9)$ and Sarsabz x NIA-Sarang (17.50, 5.00) showed increased flag leaf area over their MP and BP under $\left(\mathrm{T}_{2}\right)$. Elshafei et al. [25] observed the changes in the chlorophyll content and flag leaf senescence of wheat genotypes under water stress conditions.

Table3. Heterosis of $F_{1}$ wheat hybrids on leaf chlorophyll content and flag leaf area under water stress treatments

\begin{tabular}{|c|c|c|c|c|c|c|c|c|c|c|c|c|}
\hline \multirow{3}{*}{ Genotypes } & \multicolumn{6}{|c|}{ Leaf chlorophyll content } & \multicolumn{6}{|c|}{ Flag leaf area } \\
\hline & \multicolumn{2}{|c|}{$\begin{array}{c}\mathrm{T}_{0} \text { (Control) } \\
\text { I/D \% } \\
\end{array}$} & \multicolumn{2}{|c|}{$\begin{array}{c}\mathbf{T}_{1} \\
\text { I/D \% } \\
\end{array}$} & \multicolumn{2}{|c|}{$\begin{array}{c}\mathbf{T}_{2} \\
\text { I/D \% }\end{array}$} & \multicolumn{2}{|c|}{$\begin{array}{c}\mathbf{T}_{0} \\
\mathbf{I} / \mathbf{D} \%\end{array}$} & \multicolumn{2}{|c|}{$\begin{array}{c}\mathbf{T}_{1} \\
\mathbf{I} / \mathbf{D} \% \\
\end{array}$} & \multicolumn{2}{|c|}{$\begin{array}{c}\mathbf{T}_{2} \\
\mathbf{I} / \mathbf{D} \% \\
\end{array}$} \\
\hline & MP & BP & MP & BP & MP & BP & MP & BP & MP & BP & MP & BP \\
\hline $\mathrm{V} 1$ & 0.35 & -0.60 & 1.50 & 1.40 & -0.45 & -1.00 & 36.75 & 8.30 & 19.30 & -41.30 & 65.40 & 53.60 \\
\hline $\mathrm{V} 2$ & 4.35 & 3.80 & 5.80 & 4.30 & -2.40 & -3.70 & -44.45 & -103.6 & 33.15 & -34.20 & 45.20 & -35.0 \\
\hline V3 & -3.35 & -5.70 & -0.20 & -0.80 & 0.00 & -1.10 & -51.15 & -63.10 & -115.10 & -143.60 & -68.10 & -68.80 \\
\hline $\mathrm{V} 4$ & 9.10 & 8.40 & 2.35 & -0.30 & 2.80 & 0.80 & 19.45 & 12.8 & -90.75 & -127.60 & -39.45 & -66.30 \\
\hline V5 & 7.50 & 6.00 & 4.80 & 3.40 & 3.05 & 1.20 & 37.80 & 7.10 & 81.45 & 74.00 & 76.20 & 7.80 \\
\hline V6 & 3.70 & 2.30 & 7.90 & 7.40 & 6.15 & 4.50 & 76.90 & 60.40 & 82.4 & 59.30 & 17.50 & 5.00 \\
\hline V7 & 4.75 & 4.50 & -1.55 & -4.10 & -3.15 & 4.60 & 50.70 & 15.60 & 45.45 & 21.70 & 28.95 & 13.90 \\
\hline V8 & 11.30 & 8.40 & 1.80 & 0.90 & 3.50 & 3.30 & 117.10 & 69.90 & 120.65 & 81.80 & 116.80 & 35.90 \\
\hline V9 & 2.75 & 1.50 & 4.95 & 3.80 & 6.80 & 3.50 & 59.40 & -6.40 & 52.3 & 21.80 & 89.65 & 36.30 \\
\hline V10 & 8.15 & 6.50 & -1.25 & -3.30 & 3.00 & -0.10 & 73.00 & 55.30 & 88.95 & 80.60 & 26.15 & -1.40 \\
\hline
\end{tabular}

\section{Grain filling period}

As shown in (Table 4) under normal watering $\left(\mathrm{T}_{0}\right)$ except $\mathrm{F}_{1}$ wheat hybrid Sarsabz x Kiran-
$95(8.60,7.90)$, all the wheat hybrids revealed a decreased grain filling period. Shortest grain filling period was noted in TJ-83 x 
Kiran-95 (-11.65, -13.00) as compared to TD-1 xKiran-95 (-10.85, -11.40), NIASarang x Kiran-95 (-7.90, -10.40), T-83 x NIA-Sarang (-6.65, -7.80), TJ-83 x TD-1 ($6.50,-8.4)$, TD-1 x NIA-Sarang (-4.15, 7.20), T-83 x Sarsabz $(-4.45,-5.10)$ and Sarsabz x NIA-Sarang (-0.80, -2.60). In addition, except wheat hybrid NIA-Sarang $\mathrm{x}$ Kiran-95 $(9.15,6.90)$ and TJ-83 x Sarsabz $(0.30,0.20)$, all the hybrids showed decreased grain filling period $\left(\mathrm{T}_{1}\right)$. Shortest grain filling period against all hybrids was noted in Sarsabz x NIA-Sarang (-9.45, 11.80) followed by Sarsabz x TD-1 and TD1 x NIA-Sarang (-8.75,-10.3), TJ-83 x NIASarang (-6.75, -9.00), Sarsabz x Kiran-95 (3.80, -4.50), TD-1 x Kiran-95 (-3.65, -5.90), TJ-83 x TD-I $(-2.75,-4.20)$ and TJ-83 x Kiran-95 (-1.00, -1.80). According to results of (Table 4), all of wheat hybrids showed decreased performance over MP as well as BP under water stress from anthesis to maturity $\left(\mathrm{T}_{2}\right)$. The greatest decline in grain filling period was noted in $\mathrm{F}_{1}$ wheat hybrid TJ-83 x Kiran-95 (-17.45, -25.60) as compared to NIA-Sarang x Kiran-95 (-12.90, -21.20), TJ-83 x NIA-Sarang (-11.15, 11.30), TJ-83 x Sarsabz (-10.55, -10.70), TDI x Kiran-95 (-8.70, -16.40), TJ-83 x TD-I (7.75, -8.20), TD-1 x NIA-Sarang (-5.90, 6.50), Sarsabz x NIA-Sarang (-3.10, 3.40), Sarsabz x Kiran-95 (-3.00, -11.00) and Sarsabz x TD-I (-1.30, -1.60) $\left(\mathrm{T}_{2}\right)$. Farooq et al. [26] revealed that drought obstructs wheat performance at all growing stages; it is more serious at the time of flowering and grainfilling periods which may possibly low yield.

\section{Tillers plant ${ }^{-1}$}

According to (Table 4) the data revealed that six $F_{1}$ wheat hybrids evidently enhanced tillers plant ${ }^{-1}$ against their MP and BP as compared to other wheat hybrids. Great number of tillers plant ${ }^{-1}$ was noted in TJ-83 x TD-I $(4.90,4.20)$ followed by TJ-83 x Kiran95 (4.50 x 3.00), Sarsabz and TD-I (4.40, 4.20), TD-1 x NIA-Sarang (3.80, 2.00), TJ-
83 x Sarsabz $(3.70,3.20)$ and TD-1 x Kiran$95(2.50,0.30)$ under normal watering $\left(\mathrm{T}_{0}\right)$. Underwater stress from tillering to maturity $\left(\mathrm{T}_{1}\right), \mathrm{F}_{1}$ wheat hybrid TJ-83 x Kiran-95 (2.05, 1.00) was noted as a great tillering hybrid followed by Sarsabz x NIA-Sarang (1.80, 0.50), NIA-Sarang x Kiran-95 (1.2, 1.2), TJ83 x NIA-Sarang (1.1, 0.4), TD-1 x NIASarang $(0.85,0.20)$ and Sarsabz x Kiran-95 $(0.35,0.30)$ over their MP and BP. The results revealed that the maximum tillers plant $^{-1}$ showed by $F_{1}$ hybrid TD-1 x Kiran-95 $(2.30,2.20)$ followed by TD-1 x NIA-Sarang (2.00, 2.00), TJ-83 x NIA-Sarang (1.85, $0.40)$, Sarsabz x NIA-Sarang $(1.45,1.40)$ and Sarsabz x Kiran (1.20, 0.80) under $\left(\mathrm{T}_{2}\right)$. Destro et al. [27] revealed that tiller grain yield contributed little to the total wheat yield under water stress conditions.

\section{Harvest index}

The (Table 5) revealed that harvest index under normal watering $\left(\mathrm{T}_{0}\right)$ was noted increased $\mathrm{inF}_{1}$ wheat hybrids over MP as well as BP.Greatly increased harvest index was noted in TJ-83 x Sarsabz $(9.50,8.00)$ as compared to TD-I x NIA-Sarang (8.00, 7.00), Sarsabz x TD-I (7.00, 5.00), TJ-83 x TD-I and NIA-Sarang x Kiran-95 (6.50, 6.00), Sarsabz x NIA-Sarang (6.00, 5.00), TJ-83 x Kiran-95 (4.00, 4.00), TJ-83 x NIA-Sarang and TD-I x Kiran-95 $(2.50,2.00)$ over MP as well as BP. The $\mathrm{F}_{1}$ wheat hybrids evidently increased MP as well as BP under $\left(\mathrm{T}_{1}\right)$ for harvest index. TD-I x NIA-Sarang (0.13, $0.13)$, showed great increase in harvest index, followed by TD-1 x Kiran-95 (0.13, 0.12), TJ-83 x NIA-Sarang $(0.12,0.11)$ and Sarsabzx Kiran-95 $(0.12,0.08)$. The greatest harvest index was noted in TJ-83 x TD-I $(0.13,0.11)$ as compared to TJ-83 x NIASarang $(0.11,0.10)$ and Sarsabz x Kiran-95 $(0.10,0.07)$ respectively over their MP as well as BP. Erice et al. [28] revealed that the under optimal water availability conditions only the wheat plants with higher harvest index correlated to enhanced $\mathrm{CO} 2$ which may 
resulting enhanced plant growth under water stress conditions.

Table 4. Heterosis of $F_{1}$ wheat hybrids on grain filling period and tillers plant ${ }^{-1}$ under water treatments

\begin{tabular}{|c|c|c|c|c|c|c|c|c|c|c|c|c|}
\hline \multirow{3}{*}{ Genotypes } & \multicolumn{6}{|c|}{ Grain filling period } & \multicolumn{6}{|c|}{ Tillers plant $^{-1}$} \\
\hline & \multicolumn{2}{|c|}{$\begin{array}{c}\text { To }_{0} \text { (Control) } \\
\text { I/D \% }\end{array}$} & \multicolumn{2}{|c|}{$\begin{array}{c}\mathbf{T}_{1} \\
\text { I/D \% } \\
\end{array}$} & \multicolumn{2}{|c|}{$\begin{array}{c}\mathrm{T}_{2} \\
\mathrm{I} / \mathrm{D} \%\end{array}$} & \multicolumn{2}{|c|}{$\begin{array}{c}\mathrm{T}_{0} \\
\text { I/D } \%\end{array}$} & \multicolumn{2}{|c|}{$\begin{array}{c}\mathrm{T}_{1} \\
\mathrm{I} / \mathrm{D} \% \\
\end{array}$} & \multicolumn{2}{|c|}{$\begin{array}{c}\mathbf{T}_{2} \\
\text { I/D \% }\end{array}$} \\
\hline & MP & $\mathbf{B P}$ & MP & BP & MP & BP & MP & BP & MP & BP & MP & $\mathbf{B P}$ \\
\hline V1 & -4.45 & -5.1 & 0.3 & 0.2 & -10.55 & -10.7 & 3.7 & 3.2 & -0.2 & -1.2 & 0.7 & -0.6 \\
\hline $\mathrm{V} 2$ & -6.5 & -8.4 & -2.75 & -4.2 & -7.75 & -8.2 & 4.9 & 4.2 & -0.05 & -0.1 & -0.65 & -0.8 \\
\hline V3 & -6.65 & -7.8 & -6.75 & -9.0 & -11.15 & -11.3 & 0.3 & -0.8 & 1.1 & 0.4 & 1.85 & 0.4 \\
\hline V4 & -11.7 & -13 & -1.0 & -1.8 & -17.45 & -25.6 & 4.5 & 3.0 & 2.05 & 1.0 & -0.85 & -1.8 \\
\hline V5 & 0.45 & -0.8 & -8.75 & -10.3 & -1.3 & -1.6 & 4.4 & 4.2 & -0.65 & -1.6 & 0.25 & -1.2 \\
\hline V6 & -0.8 & -2.6 & -9.45 & -11.8 & -3.1 & -3.4 & -0.5 & -2.1 & 1.8 & 0.5 & 1.45 & 1.4 \\
\hline V7 & 8.6 & 7.9 & -3.8 & -4.5 & -3.0 & -11.0 & 0.1 & -1.9 & 0.35 & 0.3 & 1.2 & 0.8 \\
\hline V8 & -4.15 & -7.2 & -8.75 & -10.3 & -5.9 & -6.5 & 3.8 & 2.0 & 0.85 & 0.2 & 2.0 & 2.0 \\
\hline V9 & -10.9 & -11.4 & -3.65 & -5.9 & -8.7 & -16.4 & 2.5 & 0.3 & -0.6 & -1.6 & 2.3 & 2.0 \\
\hline V10 & -7.9 & -10.4 & 9.15 & 6.9 & -12.9 & -21.2 & -3.2 & -3.6 & 1.2 & 1.2 & -3.2 & -3.2 \\
\hline
\end{tabular}

\section{Biological yield plant ${ }^{-1}$}

The data in (Table 5) revealed that the four wheat hybrids were significantly increased over MP as well as BP for biological yield plant $^{-1}$ under $\left(\mathrm{T}_{0}\right)$. The maximum increased biological yield was noted in $\mathrm{F}_{1}$ wheat hybrid TJ-83 x Sarsabz $(31.20,27.06)$ followed by TD-I x Kiran-95 (27.80, 22.40), Sarsabz x Kiran-95 (19.70, 16.90), and TJ-83 x TD-I $(13.70,12.70)$ to their mid and better parent respectively. The wheat hybrid including TD-I x NIA-Sarang $(9.60,1.50)$ and Sarsabz $\mathrm{x}$ Kiran-95 (4.60, 2.30) showed increased biological yield over MP and BP under $\left(\mathrm{T}_{1}\right)$. Furthermore, five wheat hybrids over performed to both MP and BP for biological yield under $\left(\mathrm{T}_{2}\right)$. The greatest biological yield was noted in NIA-Sarang x Kiran-95 (29.05, 11.80) as compared to Sarsabz x NIA-Sarang (28.15, 23.00), TD-I x Kiran-95 (17.05, 7.60), Sarsabz x TD-I (13.35, 10.70), and TD-I x NIA-Sarang $(9.80,2.00)$ over MP and BP. $F_{1}$ wheat hybridTJ-83 x NIA-Sarang (3.90, -12.10) and Sarsabz x Kiran-95 (2.80, -9.30) were showed great biological yield over MP only under the same water stress. The rest of the F1 wheat hybrids were noted as decreased biological yield against their
$\mathrm{MP}$ as well as BP under $\left(\mathrm{T}_{2}\right)$. JohariPireivatlou [29] stated that seed and straw yield significantly reduced about $25 \%$ under water stress conditions.

\section{Relative water content}

Relative water content is an important physiological character to determine drought tolerance in wheat genotypes. The data in (Table 6), revealed that except TJ-83 x TD-I, rest of the hybrids showeds an increase to their MP and BPs for leaf relative water content under $\left(\mathrm{T}_{0}\right)$. The greater water content was noted in $\mathrm{F}_{1}$ wheat hybrid Sarsabz x NIASarang $(10.55,10.00)$, followed by Sarsabz x Kiran-95 (8.00, 7.00),TJ-83 x Sarsabz (7.95, 6.60), TD-I x Kiran-95 (7.50, 6.00), Sarsabz x TD-1 (6.5, 6.00), NIA-Sarang x Kiran-95 $(6.45,5.00)$, TJ-83 x NIA-Sarang $(2.40,0.50)$ and TD-I x NIA-Sarang $(2.35,1.30)$ which surpassed over MP and BP under normal watering (To). The maximum leaf relative water content was noted in Sarsabz x NIASarang $(10.25,10.20)$ followed by TJ-83 x NIA-Sarang (10.20, 9.30), NIA-Sarang $x$ Kiran-95 (9.15, 6.10), TD-I x Kiran-95 (8.50, 7.80), TJ-83 x Kiran-95 (8.25, 6.40), Sarsabz x Kiran-95 (6.60, 3.80), Sarsabz x TD-I (5.30, 3.20), TJ-83 x TD-I $(5.05,3.90)$, TD-I 
$\mathrm{x}$ NIA-Sarang $(4.25,2.20)$ and $\mathrm{TJ}-83 \mathrm{x}$ Sarsabz $(2.25,1.30)$ over MP and BP respectively. Meanwhile, all the $F_{1}$ wheat hybrids showed increased leaf relative water content over their mid as well as a better parent under water stress from anthesis to mature $\left(\mathrm{T}_{2}\right)$. The maximum leaf relative water content showed by hybrid TD-I x NIASarang $(8.90,6.20)$, followed by TJ-83 x Kiran-95 (8.85, 8.00), Sarsabz x NIA-Sarang (8.15, 7.40), TJ-83 x Sarsabz (7.40, 6.70), Sarsabz x TD-1 (7.25, 5.30), TJ-83 x NIA-
Sarang (4.55, 3.10), TJ-83 x TD-1 (3.55, 2.30) and TD-1 x Kiran-95 (1.60, 1.20) respectively. Wheat hybrid NIA-Sarang $\mathrm{x}$ Kiran-95 (2.00, -0.30) and Sarsabz x Kiran$95(0.85,-0.70)$ were noted as increased over their mid parent and decreased relative water content over their BP under water stress from anthesis to maturwate $\left(\mathrm{T}_{2}\right)$, respectively. Akram [30] found that the water scarcity caused lessening in leaf relative water concentrations.

Table 5. Heterotic performance of $F_{1}$ wheat hybrids on harvest index and biological yield plant $^{-1}$ under water treatments

\begin{tabular}{|c|c|c|c|c|c|c|c|c|c|c|c|c|}
\hline \multirow{3}{*}{ Genotypes } & \multicolumn{6}{|c|}{ Harvest index } & \multicolumn{6}{|c|}{ Biological yield plant ${ }^{-1}$} \\
\hline & \multicolumn{2}{|c|}{$\begin{array}{c}\mathrm{T}_{0} \\
\text { (Control) } \\
\text { I/D \% } \\
\end{array}$} & \multicolumn{2}{|c|}{$\begin{array}{c}\mathbf{T}_{1} \\
\mathbf{I} / \mathbf{D} \%\end{array}$} & \multicolumn{2}{|c|}{$\begin{array}{c}T_{2} \\
\mathbf{I} / \mathbf{D} \%\end{array}$} & \multicolumn{2}{|c|}{$\begin{array}{c}\mathbf{T}_{0} \\
\mathbf{I} / \mathbf{D} \%\end{array}$} & \multicolumn{2}{|c|}{$\begin{array}{c}\mathbf{T}_{1} \\
\mathbf{I} / \mathbf{D} \%\end{array}$} & \multicolumn{2}{|c|}{$\begin{array}{c}\mathbf{T}_{2} \\
\mathbf{I} / \mathbf{D} \%\end{array}$} \\
\hline & MP & $\mathbf{B P}$ & MP & $\mathbf{B P}$ & MP & $\mathbf{B P}$ & MP & $\mathbf{B P}$ & MP & $\mathbf{B P}$ & MP & BP \\
\hline V1 & 9.5 & 8.0 & 0.095 & 0.08 & 0.085 & 0.08 & 31.2 & 27.6 & -15.4 & -17.8 & -3.95 & -14.8 \\
\hline $\mathrm{V} 2$ & 6.5 & 6.0 & 0.06 & 0.05 & 0.13 & 0.11 & 13.7 & 12.7 & 7.8 & -1.5 & -28.7 & -36.9 \\
\hline V3 & 2.5 & 2.0 & 0.12 & 0.11 & 0.11 & 0.1 & -30.05 & -47.5 & -13.4 & -14.6 & 3.9 & -12.1 \\
\hline V4 & 4.0 & 4.0 & 0.04 & 0.02 & 0.07 & 0.05 & -4.0 & -10.4 & -7.7 & -11.4 & -4.05 & -5.3 \\
\hline V5 & 7.0 & 5.0 & 0.095 & 0.07 & 0.055 & 0.03 & -8.1 & -10.7 & -5.2 & -12.1 & 13.35 & 10.7 \\
\hline V6 & 6.0 & 5.0 & 0.105 & 0.08 & 0.075 & 0.06 & 1.25 & -12.6 & 0.5 & -6.4 & 28.15 & 23 \\
\hline V7 & 6.5 & 5.0 & 0.115 & 0.08 & 0.095 & 0.07 & 19.7 & 16.9 & 4.6 & 2.3 & 2.8 & -9.3 \\
\hline V8 & 8.0 & 7.0 & 0.13 & 0.13 & 0.07 & 0.06 & -9.35 & -25.8 & 9.6 & 1.5 & 9.8 & 2.0 \\
\hline V9 & 2.5 & 2.0 & 0.13 & 0.12 & 0.03 & 0.03 & 27.8 & 22.4 & 0.0 & $\begin{array}{l}-5.6 \\
\end{array}$ & 17.05 & 7.6 \\
\hline V10 & 6.5 & 6.0 & 0.05 & 0.04 & 0.05 & 0.04 & 5.35 & -5.7 & 1.7 & $\begin{array}{l}-0.8 \\
\end{array}$ & 29.05 & 11.8 \\
\hline
\end{tabular}

\section{Leaf proline content}

The results in (Table 6) revealed that in normal watering $\left(\mathrm{T}_{0}\right)$, wheat hybrid TJ-83 x TD-I showed high proline concentration (0.40, 0.30) over MP and BP as compared with TJ-83 x NIA-Sarang $(0.35,0.20)$ and TJ-83 x Kiran-95 (0.25, 0.00), respectively. Increased proline content over the MP was attained by $F_{1}$ hybrid Sarsabz x Kiran-95 $(0.15,-0.20)$ only. However, the rest of the wheat hybrids showed a decrease in leaf proline against MP and BP under the same watering $\left(\mathrm{T}_{0}\right)$. Great proline content was noted in Sarsabz x Kiran-95 (0.80, 0.70) as compared to Sarsabz x NIA-Sarang (0.70, 0.40), Sarsabz x TD-I (0.60, 0.20), NIASarang x Kiran-95 (0.50, 0.30), TJ-83 x
Kiran-95 (0.45, 0.20), TD-I x Kiran-95 (0.40, 0.10), TJ-83 x TD-I $(0.35,0.20)$, TJ-83 x Sarsabz $(0.35,0.00)$ and TD-1 x NIA-Sarang $(0.30,0.20)$ respectively against $\mathrm{MP}$ and $\mathrm{BP}$ under water stress from tillering to maturity $\left(\mathrm{T}_{1}\right)$. Only a single wheat hybrid TJ-83 x NIA-Sarang showed decreased proline content $(-0.35,-0.40)$ under the same water stress. It was observed that the $F_{1}$ wheat hybrids revealed increased proline content over their MP and BP $\left(\mathrm{T}_{2}\right)$. The greater proline content was observed in wheat hybrid TJ-83 x TD-I $(0.85,0.80)$ against TJ-83 x NIA-Sarang $(0.80,0.60)$, Sarsabz x TD-I andSarsabz x NIA-Sarang (0.70, 0.50), TJ-83 x Sarsabz (0.45, 0.20), TD-I x Kiran-95 $(0.40,0.40)$, TD-I x NIA-Sarang $(0.25,0.10)$, 
Sarsabz x Kiran-95 (0.20, 0.10) and NIASarang x Kiran-95 (0.05, 0.00) respectively over both MP as well as BP under $\left(\mathrm{T}_{2}\right)$.
Johari-Pireivatlou [29] found an increase of proline content in four wheat lines under water stress conditions.

Table 6. Heterosis of $F_{1}$ wheat hybrids for relative water content and leaf proline content under water treatments

\begin{tabular}{|c|c|c|c|c|c|c|c|c|c|c|c|c|}
\hline \multirow{3}{*}{ Genotype } & \multicolumn{6}{|c|}{ Relative water content } & \multicolumn{6}{|c|}{ Leaf proline content } \\
\hline & \multicolumn{2}{|c|}{$\begin{array}{c}T_{0} \text { (Control) } \\
\text { I/D \% }\end{array}$} & \multicolumn{2}{|c|}{$\begin{array}{c}\mathbf{T}_{1} \\
\mathbf{I} / \mathbf{D} \% \\
\end{array}$} & \multicolumn{2}{|c|}{$\begin{array}{c}\mathbf{T}_{2} \\
\mathbf{I} / \mathbf{D} \%\end{array}$} & \multicolumn{2}{|c|}{$\begin{array}{c}\mathbf{T}_{0} \\
\text { I/D } \%\end{array}$} & \multicolumn{2}{|c|}{$\begin{array}{c}\mathbf{T}_{1} \\
\text { I/D \% }\end{array}$} & \multicolumn{2}{|c|}{$\begin{array}{c}\mathbf{T}_{2} \\
\mathbf{I} / \mathbf{D} \%\end{array}$} \\
\hline & MP & BP & MP & BP & MP & $\mathbf{B P}$ & MP & BP & MP & $\mathbf{B P}$ & MP & $\mathbf{B P}$ \\
\hline V1 & 7.95 & 6.6 & 2.25 & 1.3 & 7.4 & 6.7 & -0.5 & -0.6 & 0.35 & 0.0 & 0.45 & 0.20 \\
\hline V2 & -0.75 & -1.6 & 5.05 & 3.9 & 3.55 & 2.3 & 0.4 & 0.3 & 0.25 & 0.2 & 0.85 & 0.80 \\
\hline V3 & 2.4 & 0.5 & .10 .2 & 9.3 & 4.55 & 3.1 & 0.35 & 0.2 & -0.35 & -0.4 & 0.80 & 0.60 \\
\hline V4 & 5.65 & 3.3 & 8.25 & 6.4 & 8.85 & 8.0 & 0.25 & 0.0 & 0.45 & 0.2 & 0.55 & 0.40 \\
\hline V5 & 6.5 & 6.0 & 5.3 & 3.2 & 7.25 & 5.3 & -0.1 & -0.3 & 0.6 & 0.2 & 0.70 & 0.50 \\
\hline V6 & 10.55 & 10.0 & 10.25 & 10.2 & 8.15 & 7.4 & -0.25 & -0.5 & 0.7 & 0.4 & 0.70 & 0.50 \\
\hline V7 & 8.0 & 7.0 & 6.6 & 3.8 & 0.85 & -0.7 & 0.15 & -0.2 & 0.8 & 0.7 & 0.20 & 0.10 \\
\hline V8 & 2.35 & 1.3 & 4.25 & 2.2 & 8.9 & 6.2 & 0.05 & 0.0 & 0.3 & 0.2 & 0.25 & 0.10 \\
\hline V9 & 7.5 & 6.0 & 8.5 & 7.8 & 1.6 & 1.2 & -0.15 & -0.3 & 0.4 & 0.1 & 0.40 & 0.40 \\
\hline V10 & 6.45 & 5.0 & 9.15 & 6.1 & 2.0 & $\begin{array}{l}-0.3 \\
\end{array}$ & -0.2 & $\begin{array}{l}-0.3 \\
\end{array}$ & 0.5 & 0.3 & 0.05 & 0.0 \\
\hline
\end{tabular}

\section{Days to $50 \%$ flowering}

The (Table 7) showed that for days to $50 \%$ flowering, F1wheat hybrid TD-1 x NIASarang attained minimum days to $50 \%$ flowering under normal watering as compared to TJ-83 x Kiran-95 (-1.95, -4.30), Sarsabz x Kiran-95 (-1.05, -5.2) and NIASarang x Kiran-95 (-0.65, -4.2) over their MP and BPs under $\left(\mathrm{T}_{0}\right)$. The $\mathrm{F}_{1}$ wheat hybrid NIA-Sarang x Kiran-95 (-3.75, -6.00), followed by TJ-83 x Sarsabz $(-2.85,-4.50)$, Sarsabz x Kiran-95 (-1.60, -2.60) which showed early flowering against their MP and $\mathrm{BP}$ under Extreme water stress $\left(\mathrm{T}_{1}\right) . \mathrm{F}_{1}$ wheat hybrid TJ-83 x Kiran-95 (-3.70, -5.20), TJ-83 x Sarsabz (-2.5 x -5.5) and Sarsabz x Kiran$95(-2.50,-4.00)$ showed early $50 \%$ flowering followed by their MP and BP under $\left(\mathrm{T}_{2}\right)$.

\section{Days to $90 \%$ maturity}

According to result in (Table 7), $\mathrm{F}_{1}$ wheat hybrid TJ-83 x Kiran-95 (-13.60, -14.60) was noted as earliest maturing as compared to other F1 wheat hybrids against their mid and better parent under normal watering $\left(\mathrm{T}_{0}\right)$ followed by NIA-Sarang x Kiran-95 (-8.55, 9.60), TD-1 x NIA-Sarang (-6.35 -7.30), TD-
1 x Kiran-95 (-4.5, -6.5), TJ-83 x TD-1 ($3.60,-2.60)$ and TJ-83 x Sarsabz (-0.20, 2.60) which revealed reduced days to $90 \%$ maturity against their MP and BPs under $\left(\mathrm{T}_{0}\right)$. The maximum decreased days to $90 \%$ maturity was noted in Sarsabz x TD-1 (8.10,-9.10), Sarsabz x Kiran-95 (-5.40, 7.10), Sarsabz x NIA-Sarang (-4.40, -5.30), TJ-83 xSarsabz (-2.55, -4.10), TD-1 x NIASarang $(-1.20,-1.30)$ and TJ-83 x TD-1 ($0.85,-1.40)$ respectively under normal watering $\left(\mathrm{T}_{1}\right)$. The genotypes TJ-83 x Kiran95 (-21.00, -27.60) was earliest maturing wheat hybrid followed by TJ-83 x Sarsabz (12.95, -15.80), TJ-83 x NIA-Sarang (-9.50, 13.20), TJ-83 x TD-I (-5.75, -7.30) Sarsabz x Kiran-95 (-5.45, -14.90), TD-I x Kiran-95 ($5.05,-13.20)$, TD-I x NIA-Sarang $(-3.15 \mathrm{x}-$ 5.30), Sarsabz x NIA-Sarang (-0.65, -1.50) for days to $90 \%$ maturity against their MP as well as BP under parent under water stress from anthesis to maturity $\left(\mathrm{T}_{2}\right)$. Panhwar et al. [31] also noted remarkable variations in days to $50 \%$ flowering and days to $90 \%$ maturity among four wheat genotypes. 
Table 7. Heterosis of $\mathrm{F}_{1}$ wheat hybrids for days to $50 \%$ flowering and days to $90 \%$ maturity under water treatments

\begin{tabular}{|c|c|c|c|c|c|c|c|c|c|c|c|c|}
\hline \multirow{3}{*}{ Genotypes } & \multicolumn{6}{|c|}{ Days to $50 \%$ flowering } & \multicolumn{6}{|c|}{ Days to $90 \%$ maturity } \\
\hline & \multicolumn{2}{|c|}{$\begin{array}{c}\text { T}_{0} \text { (Control) } \\
\text { I/D \% }\end{array}$} & \multicolumn{2}{|c|}{$\begin{array}{c}\mathbf{T}_{1} \\
\mathbf{I} / \mathrm{D} \%\end{array}$} & \multicolumn{2}{|c|}{$\begin{array}{c}\mathrm{T}_{2} \\
\mathrm{I} / \mathrm{D} \%\end{array}$} & \multicolumn{2}{|c|}{$\begin{array}{c}\mathbf{T}_{0} \\
\mathbf{I} / \mathbf{D} \%\end{array}$} & \multicolumn{2}{|c|}{$\begin{array}{c}\mathbf{T}_{1} \\
\text { I/D } \%\end{array}$} & \multicolumn{2}{|c|}{$\begin{array}{c}\mathbf{T}_{2} \\
\mathbf{I} / \mathbf{D} \%\end{array}$} \\
\hline & MP & BP & MP & BP & MP & BP & MP & BP & MP & BP & MP & BP \\
\hline V1 & 4.3 & 2.5 & -2.85 & -4.5 & -2.5 & -5.5 & -0.2 & -2.6 & -2.55 & -4.1 & -12.95 & -15.8 \\
\hline $\mathrm{V} 2$ & 3.75 & 2.8 & 1.90 & 1.0 & 1.95 & 0.0 & -2.6 & -3.6 & -0.85 & -1.4 & -5.75 & -7.3 \\
\hline V3 & 8.2 & 7.0 & 7.0 & 5.4 & 1.65 & -1.9 & 1.45 & 1.4 & 0.35 & -0.3 & -9.5 & -13.2 \\
\hline V4 & -1.95 & -4.3 & 1.45 & 0.8 & -3.7 & -5.2 & -13.6 & -14.6 & 0.45 & 0.3 & -21 & -27.6 \\
\hline V5 & 2.35 & -0.4 & 0.65 & -1.9 & 5.95 & 4.9 & 2.8 & 1.4 & -8.1 & -9.1 & 4.7 & 3.4 \\
\hline V6 & 7.5 & 6.9 & 5.05 & 1.8 & 2.45 & 1.9 & 6.65 & 4.3 & -4.4 & -5.3 & -0.65 & -1.5 \\
\hline V7 & -1.05 & -5.2 & -1.6 & -2.6 & -2.5 & -4.0 & 7.5 & 4.1 & -5.4 & -7.1 & -5.45 & -14.9 \\
\hline V8 & -2.25 & -4.4 & 9.9 & 9.2 & 2.7 & 1.1 & -6.35 & -7.3 & -1.2 & -1.3 & -3.15 & -5.3 \\
\hline V9 & 6.3 & 4.9 & 6.45 & 4.9 & 3.45 & 3.0 & -4.5 & -6.5 & 2.8 & 2.1 & -5.05 & -13.2 \\
\hline V10 & -0.65 & -4.2 & -3.75 & -6.0 & 3.95 & 1.9 & -8.55 & -9.6 & 4.6 & 3.8 & -11.05 & $\begin{array}{l}-19.2 \\
\end{array}$ \\
\hline
\end{tabular}

\section{Grain yield plant ${ }^{-1}$}

Grain yield can be considered an important trait that may possibly be contributed by many morphological traits. Therefore, grain yield is a polygenic character. According to (Table 8), $F_{1}$ wheat hybrids showed increased grain yield over MP and BP. Hybrid NIASarang x Kiran-95 (15.40, 15-00) showed increased grain yield plant ${ }^{-1}$ against MP and BP followed by Sarsabz x Kiran-95 (16.30, 12.20), TJ-83 x TD-I (15.15, 13.80), Sarsabz x TD-I $(13.15,9.70)$, TD-I x NIA-Sarang (12.85, 11.80), Sarsabz x TD-1 (13.15, 9.70), Sarsabz x NIA-Sarang $(12.70,8.20)$ and TD1 x Kiran-95 $(10.65,10.00)$ respectively under normal watering $\left(\mathrm{T}_{0}\right)$. Meles et al. [7] found increased heterosis over both the parents for grain yield. Wheat hybrids surpassed grain yield over MP and BP under extreme water stress $\left(\mathrm{T}_{1}\right)$. Hybrid TD-I $\mathrm{x}$ NIA-Sarang $(14.90,13.80)$ showed great increased grain yield as compared to TD-I x Kiran-95 (12.90, 11.50), Sarsabz x Kiran-95 (13.35, 9.20), TJ-83 x NIA-Sarang (10.30, 8.90), Sarsabz x NIA-Sarang $(9.85,7.00)$, Sarsabz x TD-1 $(8.95,7.20)$, TJ-83 x TD-I (8.10, 7.80), TJ-83 x Sarsabz $(8.05,6.60)$. Underwater stress from anthesis to maturity $\left(\mathrm{T}_{2}\right)$, NIA-Sarang $\mathrm{x}$ Kiran-95 (35.30, 9.10), Sarsabz x Kiran-95 (11.55, 7.60), TJ-83 x NIA-Sarang $(9.75,7.00)$, and TJ-83 x
Sarsabz $(8.90,8.00)$ showed increased grain yield over MP and BP. Çifci [32] observed that the heterosis for grain yield and positive correlations with grain yield by agronomical traits.

\section{0 grain weight}

The data in (Table 8) highlighted that all wheat hybrids found an increased 1000 grain weight over their mid and better parent. While, the maximum increase of 1000 grain weight was noted in TJ-83 x Sarsabz (26.35, 24.90) as compared to NIA-Sarang x Kiran95 (15.95, 14.30), TJ-83 x Kiran-95 (13.35, 12.50), TJ-83 x TD-I (12.10, 11.90), TJ-83 x NIA-Sarang $(10.90,10.10)$ and TD-I $x$ NIASarang $(10.60,9.60)$ respectively over MP and $\mathrm{BP}$, under normal watering $\left(\mathrm{T}_{0}\right)$. As a result, all $\mathrm{F}_{1}$ wheat hybrids showed increased 1000grain weight against their MP as well as BP under $\left(\mathrm{T}_{1}\right)$. However, the greatest increase of 1000 grain weight was noted in Sarsabz x TD-I $(3.55,2.30)$ followed by NIA-Sarnag x Kiran-95 (3.40, 2.80), TD-I x NIA-Sarang $(2.25,1.30)$, TJ-83 x Kiran-95 (1.55, 0.50), Sarsabz x NIA-Sarang (0.60, $0.30)$ and TD-I x Kiran-95 $(0.45,0.10)$ over MP as well as BP respectively under $\left(\mathrm{T}_{2}\right)$. Wheat hybrid T-83 x Sarsabz (4.35, -1.00), TJ-83 x TD-1 $(0.40,-0.30)$ and TJ-83 x NIASarang $(0.15,-1.5)$ showed as increased 1000 grain weight over their MP only under same 
water stress. Johari-Pireivatlou [29] observed that the maximum high seed yield, straw yield, harvest index, and 1000 kernel weight were observed under control conditions (Non-stress).

Table 8. Heterosis of $F_{1}$ wheat hybrids on grain yield plant ${ }^{-1}$ and 1000 grain weight under water treatments

\begin{tabular}{|c|c|c|c|c|c|c|c|c|c|c|c|c|}
\hline \multirow{3}{*}{ Genotypes } & \multicolumn{6}{|c|}{ Grain yield plant $^{-1}$} & \multicolumn{6}{|c|}{1000 grain weight } \\
\hline & \multicolumn{2}{|c|}{$\begin{array}{c}\text { To }_{0} \text { (Control) } \\
\text { I/D \% }\end{array}$} & \multicolumn{2}{|c|}{$\begin{array}{c}\mathbf{T}_{1} \\
\mathbf{I} / \mathbf{D} \%\end{array}$} & \multicolumn{2}{|c|}{$\begin{array}{c}\mathbf{T}_{2} \\
\mathbf{I} / \mathbf{D} \%\end{array}$} & \multicolumn{2}{|c|}{$\begin{array}{c}\mathrm{T}_{0} \\
\mathrm{I} / \mathrm{D} \%\end{array}$} & \multicolumn{2}{|c|}{$\begin{array}{c}\mathbf{T}_{1} \\
\mathbf{I} / \mathbf{D} \%\end{array}$} & \multicolumn{2}{|c|}{$\begin{array}{c}\mathbf{T}_{2} \\
\mathbf{I} / \mathbf{D} \%\end{array}$} \\
\hline & MP & BP & MP & $\mathbf{B P}$ & MP & $\mathbf{B P}$ & MP & BP & MP & BP & MP & BP \\
\hline V1 & 6.8 & 4.7 & 8.05 & 6.6 & 8.9 & 8.0 & 26.35 & 24.9 & 4.35 & -1 & 10 & 8.5 \\
\hline V2 & 15.15 & 13.8 & 8.1 & 7.8 & 8.0 & 6.7 & 12.1 & 11.9 & 0.4 & -0.3 & 10.25 & 10.2 \\
\hline V3 & $\begin{array}{l}-0.9 \\
\end{array}$ & \begin{tabular}{|l|}
-3.3 \\
\end{tabular} & 10.3 & 8.9 & 9.75 & 7.0 & 10.9 & 10.1 & 0.15 & -1.5 & 5.15 & 3.8 \\
\hline V4 & 4.2 & 2.2 & 3.6 & 1.9 & 8.95 & 5.9 & 13.35 & 12.5 & 1.55 & 0.5 & 10.95 & 10.0 \\
\hline V5 & 13.15 & 9.7 & 8.95 & 7.2 & 8.3 & 6.1 & 4.75 & 3.1 & 3.55 & 2.3 & 10.55 & 9.1 \\
\hline V6 & 12.7 & 8.2 & 9.85 & 7 & 9.15 & 5.5 & 8.35 & 7.7 & 0.6 & 0.3 & 8.05 & 5.2 \\
\hline V7 & 16.3 & 12.2 & 12.35 & 9.2 & 11.55 & 7.6 & 8.1 & 5.8 & 2.7 & 1.8 & 9.55 & 7.1 \\
\hline V8 & 12.85 & 11.8 & 14.9 & 13.8 & 5.95 & 4.5 & 10.6 & 9.6 & 2.25 & 1.3 & 6.6 & 5.2 \\
\hline V9 & 10.65 & 10.0 & 12.9 & 11.5 & 7.75 & 6 & 9.45 & 8.8 & 0.45 & 0.1 & 13 & 12.0 \\
\hline V10 & 15.4 & 15 & 5.7 & 5.4 & 35.3 & 9.1 & 15.95 & 14.3 & 3.4 & 2.8 & 11.9 & 11.5 \\
\hline
\end{tabular}

\section{Pearson correlation coefficient}

Correlation studies are a useful tool for plant breeders to understand the relationship between yield and its related components. Correlation helps for the improvement of drought tolerant cultivars in the sense that physiological parameters might be used as indirect choice criteria to enhance grain yield under water stress environment. Results in (Table 9) indicated that highly positive significant association with grain yield was observed in biological yield plant ${ }^{-1}(0.745)$, days to $90 \%$ maturity $(0.727)$, grain yield main spike ${ }^{-1}(0.590)$, harvest index (0.747), main spike length (0.714), plant height (0.705) and tillers plant ${ }^{-1}(0.584)$. While chlorophyll content $(0.228)$, grains spike $^{-}$
${ }^{1}(0.202)$, nodes tiller ${ }^{-1}(0.288)$, spikelet spike ${ }^{1}(0.400)$ were medium positively correlated with grain yield plant ${ }^{-1}$. Highly negative correlations (-0.669) were noted between grain yield and days to $50 \%$ flowering, a medium negative correlation was noted between grain yield and 1000grain weight (0.366). A low positive correlation (0.011) was noted between grain yield and proline content. A low negative correlation coefficient (-0.071) was noticed in between flag leaf area and grain yield plant ${ }^{1}$. Zerga $e t$ al. [33] observed the positive association among growth and yield related traits in bread wheat. Also, Meles et al. [7] reported such positive correlations of grain yield with other agronomical traits. 
Table 9. Pearson correlation coefficient among some morpho-physiological traits of wheat genotypes

\begin{tabular}{|c|c|c|c|c|c|c|c|c|c|c|c|c|c|c|c|c|c|}
\hline Traits & BY & CL & Ds50\% & Ds90\% & FLA & GYP & GYS & GPS & HI & MPL & NPT & PH & PC & RWC & $1000 \mathrm{G}$ & SPS & TPP \\
\hline BY & 1 & & & & & & & & & & & & & & & & \\
\hline CL & -0.024 & 1 & & & & & & & & & & & & & & & \\
\hline Ds50\% & -0.393 & -0.464 & 1 & & & & & & & & & & & & & & \\
\hline Ds90\% & 0.624 & 0.305 & -0.685 & 1 & & & & & & & & & & & & & \\
\hline FLA & 0.152 & -0.294 & 0.134 & -0.070 & 1 & & & & & & & & & & & & \\
\hline GYP & 0.745 & 0.228 & -0.669 & 0.727 & -0.070 & 1 & & & & & & & & & & & \\
\hline GYS & 0.522 & 0.016 & -0.305 & 0.555 & 0.038 & 0.590 & 1 & & & & & & & & & & \\
\hline GPS & 0.116 & -0.073 & 0.012 & 0.131 & 0.045 & 0.202 & 0.425 & 1 & & & & & & & & & \\
\hline HI & 0.667 & 0.184 & -0.457 & 0.748 & 0.116 & 0.747 & 0.658 & 0.089 & 1 & & & & & & & & \\
\hline MPL & 0.504 & 0.375 & -0.786 & 0.722 & -0.002 & 0.714 & 0.462 & 0.300 & 0.610 & 1 & & & & & & & \\
\hline NPT & 0.424 & -0.165 & 0.104 & 0.224 & -0.132 & 0.288 & 0.261 & 0.131 & 0.349 & 0.054 & 1 & & & & & & \\
\hline PH & 0.658 & 0.028 & -0.473 & 0.685 & -0.074 & 0.705 & 0.610 & 0.324 & 0.711 & 0.652 & 0.400 & 1 & & & & & \\
\hline PC & -0.219 & 0.330 & -0.502 & 0.026 & -0.260 & 0.011 & -0.343 & -0.143 & 0.102 & 0.010 & -0.051 & -0.028 & 1 & & & & \\
\hline RWC & -0.169 & -0.234 & -0.403 & -0.116 & 0.344 & -0.341 & -0.111 & -0.356 & 0.028 & 0.693 & 0.251 & -0.623 & -0.752 & 1 & & & \\
\hline $1000 \mathrm{G}$ & 0.093 & 0.759 & 0.494 & -0.144 & 0.246 & -0.366 & 0.118 & -0.249 & 0.026 & -0.084 & 0.089 & 0.300 & 0.611 & 0.132 & 1 & & \\
\hline SPS & 0.349 & 0.054 & 0.324 & 0.072 & 0.652 & 0.400 & 0.007 & $\begin{array}{l}-0.46 \\
\end{array}$ & 0.234 & -0.387 & -0.202 & -0.083 & -0.179 & -0.020 & -0.171 & 1 & \\
\hline TPP & -0.092 & 0.190 & 0.040 & -0.205 & -0.331 & 0.584 & -0.207 & -0.157 & 0.045 & 0.185 & 0.713 & 0.739 & 0.192 & 0.698 & 0.016 & -0.014 & 1 \\
\hline
\end{tabular}

$\mathrm{BY}=$ Biological yield, $\mathrm{CL}=$ chlorophyll content, Ds50\%= Days to 50\% flowering, Ds90\%= Days to 90\% maturity, FLA=Flag leaf area, GYP= Grain yield per plant, GYS= Grain yield per main spike, GMnS=Grains/main spike $\mathrm{HI}=$ Harvest index, $\mathrm{MSL}=\mathrm{Main}$ spike length, NPT= Nods/main tiller, $\mathrm{PH}=$ plant height, $\mathrm{PC}=\mathrm{Prolin}$ content, RWC = Relative water content, $1000 \mathrm{G}=1000$ grain weight, $\mathrm{SPS}=$ Spikelet/spike, and TPP=Tillers per plant 


\section{Conclusion}

It is concluded that the results of the present research work had confirmed that the importance of heterosis and association of characters are as efficient criteria for the evaluation of wheat genotypes. $\mathrm{F}_{1}$ wheat hybrids showed large genetic variation under water treatments, indicating increased heterosis. It is suggested that $F_{1}$ wheat hybrid NIA-Sarang x Kiran-95, TD-1 x NIASarang and Sarsabz x Kiran-5 were observed superior with high heterosis for water stress tolerance and grain yield, therefore could be considered for breeding water stress tolerant genotypes. Farmers need to grow wheat genotypes with high grain yield and straw yield. Therefore wheat hybrids TJ-83 x Sarsabz, Sarsabz x Kiran-95, TD-1 xNIA-Sarang, NIASarang $x$ Kiran-95, and Sarsabz x NIA-Sarang showed greater performance in biological yield and production under water stress conditions.

\section{Authors' contributions}

Conceived and designed the experiments: NA Panhwar, GM Baloch \& ZA Soomro, Performed the experiments: NA Panhwar \& MA Sial, Analyzed the data: NA Panhwar, GM Baloch \& ZA Soomro, Contributed materials/ analysis/ tools: NA Panhwar, SA Panhwar \& A Afzal, Wrote the paper: NA Panhwar \& AH Lahori.

\section{Acknowledgment}

I would like to thank the Director of NIA Tandojam and their staff members for providing me laboratory facilities and experimental field for this rxperiment.

\section{References}

1. Murphy KM, Reeves PG \& Jones SS (2008). Relationship between yield and mineral nutrient concentrations in historical and modern spring wheat cultivars. Euphytica 163(3): 381-390.

2. Panhwar NA, Buriro SA, Memon AH, Panhwar SA \& Lahori AH (2021). Influence of salinity on germination and early seedling of five wheat (Triticumaestivum L.) genotypes. Pure and Appl Biol 10(4): 956961.

3. Dawson TP, Perryman AH \& Osborne TM (2016). Modelling impacts of climate change on global food security. Clim Change 134(3): 429-440.

4. Rötter RP, Tao F, Höhn JG \& Palosuo T (2015). Use of crop simulation modelling to aid ideotype design of future cereal cultivars. $J$ Exp Bot 66(12): 3463-3476.

5. Kumar J, Singh SK, Kumar LS, Anurag A, Singh SK \& Kumar M (2016). Estimates of general and specific combining ability for grain yield and other physiological characters in bread wheat under late sown condition. Res Environ Life Sci 9(7): 784-789.

6. Nybom H (2004). Comparison of different nuclear DNA markers for estimating intraspecific genetic diversity in plants. $\mathrm{Mol}$ Ecol 13(5): 1143-1155.

7. Meles B, Mohammed W \& Tsehaye Y (2017). Genetic variability, correlation and path analysis of yield and grain quality traits in bread wheat (Tritium aestivum L.) genotypes at Axum, Northern Ethiopia. $J$ Plant Breeding Crop Sci 9(10): 175-85.

8. Chimdesa O, Mohammed W \& Eticha $\mathrm{F}$ (2017). Analysis of genetic variability among breadw heat (Triticumaestivum L.) Genotypes for growth, yield and yield components in bore District, Oromia Regional State. Agri Forest Fish 6(6): 188199.

9. Kahrizi D, Maniee M, Mohammadi R \& Cheghamirza K (2010). Estimation of genetic parameters related to morpho-agronomic traits of durum wheat (Triticum turgidum var. durum). Biharean Biol 4(2): 93-97.

10. Sial MA, Laghari KA, Panhwar NA, Arain MA \& Baloch GM (2012). Genetic improvement of drought tolerance in semidwarf wheat. Sci Tech Devel 31(4): 335-340.

11. Asifa K, Soomro ZA, Mari SN, Sial MA, Baloch M \& Albokari MM (2015). Evaluation of water stress tolerance in wheat genotypes on the basis of phenological and morphological traits. Int J Biol Biotech 12(2): 261-270.

12. Bernardo L, Carletti PF, Badeck W, Rizza F, Morcia C, Ghizzoni R, Rouphael Y, Colla G, Terzi V \& Lucini L (2019). Metabolomic responses triggered by arbuscularmycorrhiza enhance tolerance to water stress in wheat cultivars. Plant Physiol Bioch 137: 203-212.

13. Lin T, Zhou C, Chen G, Yu J, Wu W, Ge Y, Liu X, Li J, Jiang X, Tang W \&Tian, Y (2020). Heterosis-associated genes confer high yield in super hybrid rice. Theor Appl Genet 133(12): 3287-3297. 
14. Moosavi SS, Abdi F, Abdollahi MR, Tahmasebi-Enferadi S \& Maleki M (2020). Phenological, morpho-physiological and proteomic responses of Triticumboeoticum to drought stress. Plant Physiol Bioch 1(156): 95-104.

15. Barrs HD \& Weatherley PE (1962). A reexamination of the relative turgidity technique for estimating water deficit in leaves. Aust J Bio Sci 15: 413-428.

16. Bouyoucos GJ (1962). Hydrometer method improved for making particle size analysis of soils. Agron J 53: 464-465.

17. Veihmeyer FJ \& Hendrickson A.H (1949). Methods of measuring field capacity and permanent wilting percentage of soils. Soil Sci 68(1): 75-94.

18. Jackson ML (1969). Mineral fraction for soils. In: Soil Chemical Analyses Advanced Course. M.L. Jackson (eds). Uni Wisconsin, Madison, WT. 100-168.

19. Kwon SI, Jang YA, Kim KH, Jung GB, Kim MK, Hwang H, Chae MJ, Hong SC, So KH, Yun SG \&Kim KR (2012). Heavy metal chemistry in soils received long-term application of organic wastes. J Agri Food Chem 1: 1-9.

20. Olsen SR \& Sommer, LE (1982). Phosphorus. In: Page, A.L. (Ed.), Methods of Soil Analysis, Part 2, 2nd edn, Agron Monogr 9, 2nd edn. ASA and ASSA, Madison WI, pp. 403-430.

21. Baloch M, Baloch AW, Siyal NA, Baloch SN, Soomro AA, Baloch SK \& Gandahi N (2016). Heterosis Analysis in F1Hybrids of Bread Wheat. Sindh Uni Res J-SURJ (Sci Ser) 48(2): 261-264.

22. Baloch MJ, Chandio IA, Arain MA, Baloch A \& Jatoi WA (2016). Effect of terminal drought stress on morpho-physiological traits of wheat genotypes. Biol Sci-PJSIR 59(3): 117-125,

23. Steel RGD \& Torrie JH (1980). Principles and procedures of statistics. A Biometrical Approach. McGraw-Hill Company, New York. pp.393, DOI: 10.2307/2530180.

24. Sharma SN, Sain RS \& Sharma RK (2003). Genetics of spike length in durum wheat. Euphytica 130(2): 155-161.
25. Elshafei AA, Saleh M, Al-Doss AA, Moustafa KA, Al-Qurainy FH \& Barakat MN (2013). Identification of new SRAP markers linked to leaf chlorophyll content, flag leaf senescence and cell membrane stability traits in wheat under water-stressed condition. Aust J Crop Sci 7(6): 887-893.

26. Farooq M, Hussain M \& Siddique KHM (2014). Drought stress in wheat during flowering and grainFilling periods. Crit Rev Plant Sci 33: 331-349.

27. Destro D, Miglioranza É, Arias CAA, Vendrame JM \& Almeida JCVD (2001). Main stem and tiller contribution to wheat cultivars yield under different irrigation regimes. Braz Arch Bio Tech 44(4): 325330.

28. Erice G, Sanz-Sáez A, Urdiain A, Araus JL, Irigoyen JJ \& Aranjuelo I (2014). Harvest index combined with impaired $\mathrm{N}$ availability constrains the responsiveness of durum wheat to elevated $\mathrm{CO}_{2}$ concentration and terminal water stress. Funct Plant Biol 41(11): 1138-1147.

29. Johari-Pireivatlou M (2010). Effect of soil water stress on yield and proline content of four wheat lines. Afr J Biotechn 9(1): 036040 .

30. Akram M (2011). Growth and yield components of wheat under water stress of different growth stages. Bangl J Agri Res 36(3): 455-468.

31. Panhwar NA, Buriro SA, Memon AH, Tunio M, Sehar NU, Panhwar SA \& Lahori AH (2020). Influence of row spacing on grain yield of winter wheat genotypes. FUUAST J Biol 10(2): 103-108.

32. Çifci EA (2012). Estimate of heterosis, correlation and path analysis for grain yield per spike and some agronomic traits on durum wheat (Triticum durum Desf). J Anim Plant Sci 22(3): 747-752.

33. Zerga K, Mekbib F and Dessalegn T (2016). Estimation of association among growth and yield related traits in bread wheat (Triticum aestivum L.) genotypes at Gurage Zone, Ethiopia. J Plant Breed Crop Sci 3(2): 123134. 